\title{
Tissue Nuclear Matrix Protein Expression 22 in Various Grades and Stages of Bladder Cancer
}

\author{
Quratulain Mati ${ }^{1}$, Samina Qamar ${ }^{1}$, Sobia Ashraf ${ }^{2}$, Muhammad Abbas Khokhar ${ }^{3}$ and Usma Arshad ${ }^{1}$ \\ ${ }^{1}$ Department of Histopathology, King Edward Medical University, Lahore, Pakistan \\ ${ }^{2}$ Department of Hematology, King Edward Medical University, Lahore, Pakistan \\ ${ }^{3}$ Department of Oncology, King Edward Medical University, Lahore, Pakistan
}

\begin{abstract}
Objective: To study the tissue nuclear matrix protein 22 (NMP22) expression in bladder carcinoma biopsy and correlate it with prognostic factors like stage and grade of bladder cancer.

Study Design: Descriptive cross-sectional study.

Place and Duration of Study: Pathology and Urology Departments, King Edward Medical University/Mayo Hospital, Lahore, Pakistan from January to October 2019.

Methodology: Data of all bladder cancer patients' biopsy blocks, presenting to histopathology section, was collected. NMP22 protein expression levels were analysed by immunohistochemical staining using a quantitative scoring method defined by staining intensity (I), and percentage of positive-stained cells (P). Data was analysed by SPSS version 22 . Chi-square test and crosstabs were used to correlate grade and stage of urothelial tumors with gender and NMP22 expression. $p \leq 0.05$ was taken as significant.

Results: A total of 95 paraffin blocks of bladder carcinoma biopsies with data were retrieved for the study. NMP22 was expressed immunohistochemically in 87/95(91.6\%) of cases and was negative in 8/95(8.4\%) of cancers. Correlation of intensity of NMP22 staining with histologic grade showed that $58.3 \%$ of high grade BC expressed NMP22 strongly, $23.6 \%$ moderately and $11.1 \%$ weakly. In low grade tumors, no biopsy (0/23) expressed strong expression. Moderate intensity in $8.69 \%$ and weak in $78.26 \%$ of tumors $(p<0.001)$. Intensity of staining with stage of tumor association revealed that $40.8 \%$ of stage I tumor showed strong staining, $16.9 \%$ moderate, and $32.3 \%$ weak staining pattern. In stage II tumors $54.1 \%$ showed strong, $29.1 \%$ moderate, and $12.5 \%$ weak NMP 22 expression. $(p=0.148)$.

Conclusion: NMP 22 is highly expressed in urinary bladder carcinoma and in male patients. It has strong association with grade and weak association with stage of tumor. Immunohistochemical expression of this protein can help in better detection and prognostic prediction of bladder carcinoma.
\end{abstract}

Key Words: Bladder carcinoma, Grade, Immunohistochemistry, NMP22, Stage.

How to cite this article: Mati Q, Qamar S, Ashraf S, Khokhar MA, Arshad U. Tissue Nuclear Matrix Protein Expression 22 in Various Grades and Stages of Bladder Cancer. J Coll Physicians Surg Pak 2020; 30(12):1321-1325.

\section{INTRODUCTION}

Urinary bladder cancer (BC) is the fourth most common cancer worldwide. ${ }^{1}$ In Pakistan, due to rapid growth of population and aging, bladder cancer is amongst the top ten frequent cancers as urinary bladder carcinoma occurs in $50-80$ years of life. ${ }^{2}$ If the tumor has invaded bladder muscle wall, the 5 -year survival rate is $69 \%$. If the cancer extends through the bladder to the surrounding tissues, lymph nodes or adjacent organs, the 5year survival rate is $35 \%$.

Correspondence to: Dr. Samina Qamar, Department of Histopathology, King Edward Medical University, Lahore, Pakistan

E-mail: samnir3@gmail.com

Received: January 22, 2020; Revised: April 08, 2020;

Accepted: May 12, 2020

DOI: https://doi.org/10.29271/jcpsp.2020.12.1321
If the cancer has spread to distant parts of the body, the 5-year survival rate is $5 \%$. About $4 \%$ of people are diagnosed with this stage. ${ }^{3}$ Risk factors for $\mathrm{BC}$ are smoking, infections, prior radiation and exposure to certain chemicals like benzidine and aromatic amines (also called xenobiotics) found in paints, leather industry, and hair dyes. ${ }^{4}$ Mainstay of diagnosis is cystoscopic biopsy and urine cytology. Newer noninvasive tests that bind urinary proteins like nuclear matrix protein 22 (NMP22), carcinoembryonic antigen and human complement factor $\mathrm{H}$ protein are also available. ${ }^{5}$ In some people, genes like GST (glutathionetransferase) and NAT (N-acetyl transferase) are genetically altered, that do not effectively break down toxic chemicals excreted in urine and are called slow acetylators (SA). These harmful chemicals form toxic electrophiles in urinary bladder of SAand lead to cancer development. ${ }^{6}$

NMP22 is a protein located in mitotic spindle of nucleus of the cell. It allows cell replications after microtubule assembly and 
fragmentation of genome. Its response is enhanced in presence of xenobiotics.

Its main function includes DNA organisation, stabilisation, organisation of gene complexes and RNA synthesis. NMP22 and BLCA-4 (bladder cancer-4) are two main nuclear proteins involved in bladder cancer initiation and propagation. ${ }^{7} \mathrm{NMP} 22$ is released from apoptotic cells and its concentration rises in urine of bladder cancer patients. It is considered to be most reliable, noninvasive, highly sensitive but poorly specific marker of malignancy in voided urine. ${ }^{8}$

This study was designed to study of tissue NMP22 expression in terms of prognostic factors, like stage and grade of bladder cancer.

\section{METHODOLOGY}

It was a descriptive cross-sectional study, conducted at the Pathology Department in collaboration with the Urology Department, King Edward Medical University/Mayo Hospital, Lahore, Pakistan. Data of all bladder cancer patients' biopsy blocks, presenting to histopathology section for reporting, was collected retrospectively from January to October 2019 with the help of non-probability consecutive sampling. Patients were contacted to obtain informed consents for participation in the study.

Patients who did not give consent or lost to follow-up were excluded from study population. All inadequate/insufficient tissue biopsies, and patients, who underwent chemo/radiotherapy prior to biopsy, recurrent tumor biopsies and with urinary tractinfections, were also exempted.

Data of all biopsies of bladder tumors from January to October 2019 were collected and noted. Clinical/demographic findings of patients were noted from charts of patients in Urology Ward by concerned urologist after taking informed consents from patients. Hematoxylin-eosin (HE) stained slides were prepared after processing and cases were graded according to WHO 2010 grading system. TNM system was used for pathological staging. ${ }^{9}$ Staining was performed with a specific antibody against NMP22 (1:10000, ab108600; Abcam) at room temperature for 60 mins using a standard protocol on the Bond-Max automated IHC stainer (Leica Biosystems, Global). A bond polymer refine detection system (Vision BioSystems, Melbourne, Australia) was used to reduce nonspecific staining. Immunohistochemical staining was evaluated using a quantitative scoring method defined by two parameters: staining intensity (I), and percentage of positive-stained cells (P). For staining intensity, a score of 0 represents no staining; whereas, scores of 1 -weak, 2 moderate and 3- represent strong staining. The final score was obtained by multiplying the intensity (I) by the percentage of positive-stained cell (P). NMP22 protein expression levels in BC tissue and adjacent normal cells were categorised into four groups, depending upon number of cells counted as no nuclear stain-negative staining, score 0-99; weak staining; score 100-199-moderate; and strong staining, score $\geq 200$. Method used to detect was heat shock wave protein E1/HSPE1, since no study previously done on immunohistochemical expression of
NMP22. ${ }^{10}$ All clinical and histopathological findings were noted in a predesigned proforma.

Data was analysed by SPSS version 22. Quantitative measure, like age, was expressed in terms of mean and standard deviation. Qualitative data were expressed as frequencies along with percentages. Chi-square test and crosstabs were used to study correlation between qualitative measures, like grade and stage of urothelial tumors with gender and NMP22 expression. $\mathrm{P} \leq 0.05$ was taken as significant.

\section{RESULTS}

A total of 95 paraffin blocks of bladder carcinoma biopsies with data were retrieved for the study. Age of these patients varied from $30-83$ years with a mean value of $59.8 \pm 12.63$ years. Eighty-three (87.4\%) were males and 12 (12.6\%) were females. Out of $95,23(24.2 \%)$ were low grade tumors and $72(75.8 \%)$ were high grade papillary urothelial cancer (bladder carcinoma). Seventy-one (74.7\%) patients were diagnosed of stage I (T1) and $24(25.3 \%)$ were in stage II (T2) cancers. NMP22 was expressed immunohistochemically in 87 (91.6\%) of the cases and was negative in $8(8.4 \%)$ of cancers. Strong intensity of staining was observed in 42 (44.2\%), moderate in 19 (20\%), weak in $26(27.4 \%)$ and none in 8 ( $8.4 \%$ ) of cases. (Figures 1-4).

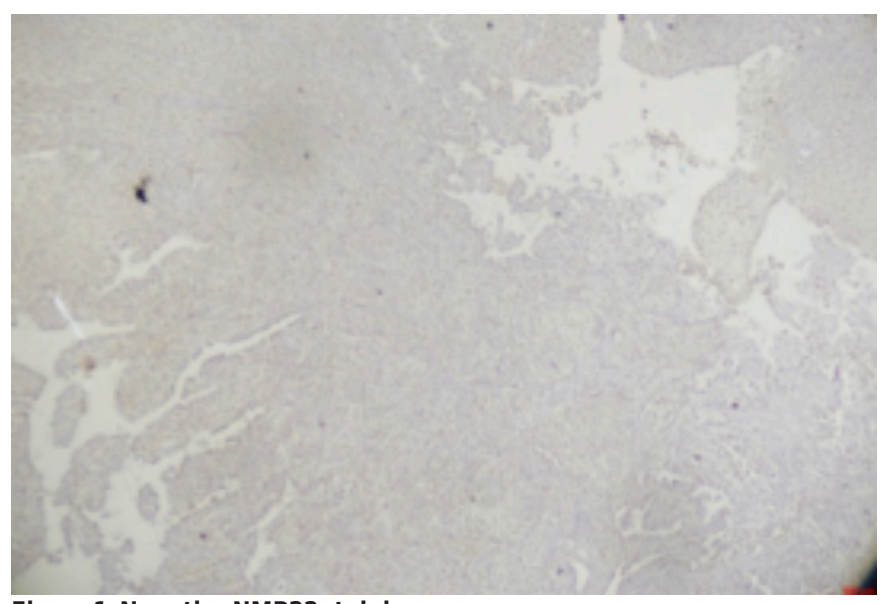

Figure 1: Negative NMP22 staining.

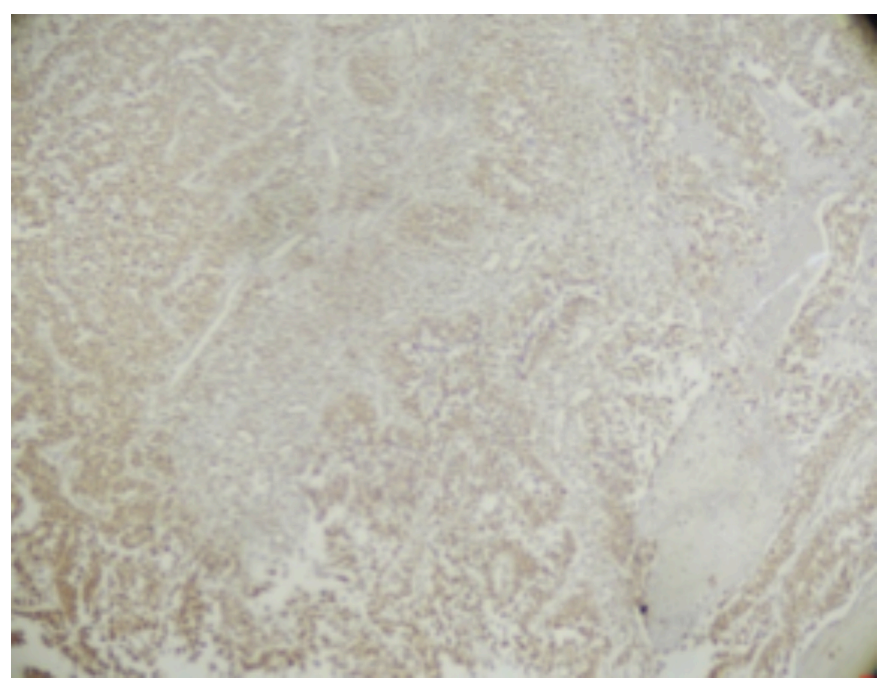

Figure 2: Weakstaining. 


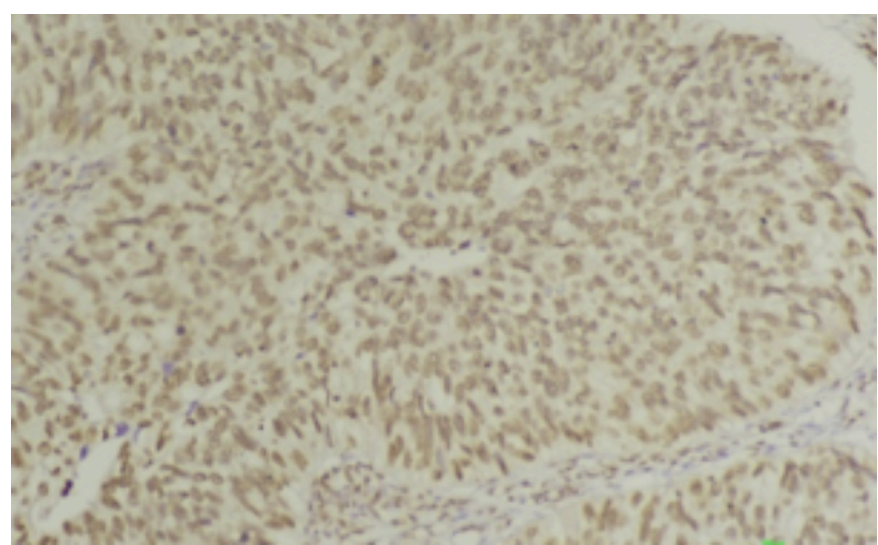

Figure 3: Moderate staining.

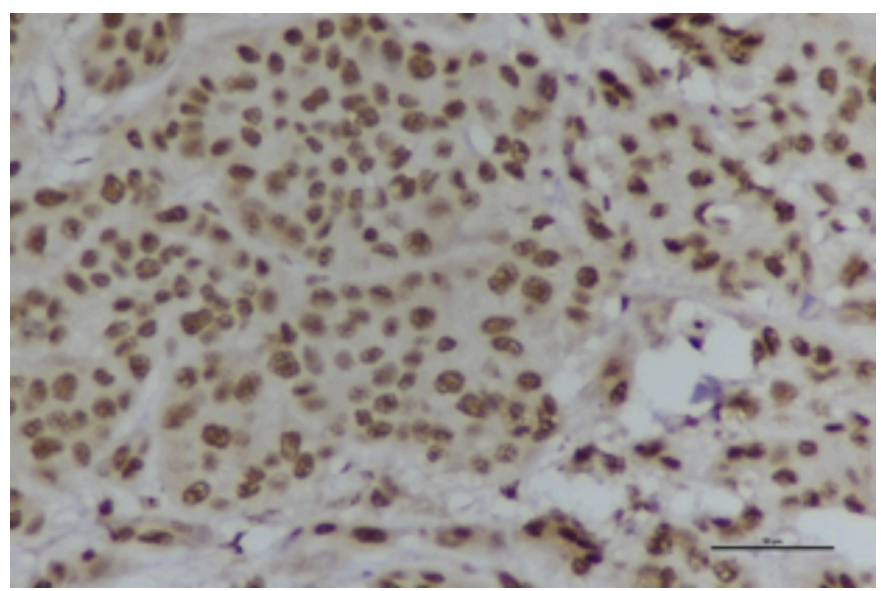

Figure 4: Strong positive NMP22.

Out of $23(24.2 \%)$ low grade tumors, 17 (73.9\%) were males and 6 (26\%) were females. Out of $72 / 95$ (75.8\%) high grade tumors $66 / 72(91.6 \%)$ were seen in males and $6 / 72(8.3 \%)$ were seen in females $(p=0.026)$. Stage I tumors were in $71 / 95(74.7 \%)$. Sixtyone $/ 71(85.9 \%)$ males and $10 / 71(14.08 \%)$ females presented with stage I tumors, and 22/24 (91.6\%) males and 2/24 (8.3\%) females were at stage $I I,(p=0.463)$. A high proportion, i.e. $92.8 \%$ (77/83) biopsies in males and 83.3\% (10/12) biopsies in female expressed NMP22 immunomarker. Association of NMP22 expression with gender was such that 22/26 (84.6\%) males and 4/26 (15.3\%) females were weak positive for NMP22. 18/19 (94.7\%) exhibited moderate positivity in males and $1 / 19(5.26 \%)$ in females. Strong expression was noted in $37 / 42$ (88.1\%) males and $5 / 42(11.9 \%)$ females. Eight out of 95 cases were negative out of which six $(75 \%)$ were males and two $(25 \%)$ were females, $(p=0.524)$.

Correlation of intensity of NMP22 staining with histologic grade showed that $58.3 \%(42 / 72)$ of high grade BC expressed NMP22 strongly, 23.6\% (17/72) moderately and $11.1 \%$ (8/72) weakly. In low grade tumors no biopsy (0/23) expressed strong expression. Moderate intensity was seen in $8.69 \%$ (2/23) and weak in $78.26 \%$ $(18 / 23)$ of tumors $(p<0.001)$. Intensity of staining with stage of tumor association revealed that $40.8 \%$ (29/71) of stage I tumor showed strong, $16.9 \%$ (12/71) showed moderate and $32.3 \%$ (23/71) showed weak staining pattern. In stage II tumors, $54.2 \%$ (13/24) showed strong, $29.2 \%$ (7/24) showed moderate and $12.5 \%(3 / 24)$ showed weak NMP 22 expression, $(p=0.148)$.

\section{DISCUSSION}

Many survival-related and stage specific genes undergo structural variations to promote evolution of bladder cancer. ${ }^{1}$ Black race and older age at diagnosis are important predictors of death. ${ }^{4}$ NMP binds to DNA sequences involved in chromosomal replication, transcription and interaction with isomerases. Urinary levels of NMP22 are raised in cancer patients; however, its disadvantage is the possibility of false positive results due to NMP22 release from dead urothelial cells in bladder infections. ${ }^{11}$ Since there is high potential for targeted therapies and immunotherapy in urothelial tumors, there is a continuous search for molecular predictive and prognostic markers. ${ }^{6}$ NMP22 urine cytology alone has modest specificity and low sensitivity for cancer detection. ${ }^{11}$ Combination of FISH (Fluorescence in situ hybridisation) and NMP22 can increase the sensitivity and specificity of bladder cancer (BC) detection; but it can increase the cost of management the disease ${ }^{12}$.

Many researchers have proposed association of NMP22 cytology with grade and stage of bladder carcinoma but no study has emphasised cancer detection and management with the help of immunohistochemistry, which is cheaper than FISH and cytology techniques.

This study included biopsy samples of 95 patients with mean age of $59.8 \pm 12.63$ years with majority of male patients. This was similar to other international data presented by many other studies showing median age of 58 years and males being more commonly affected by it. ${ }^{13-15}$ In this cohort, 23 (24.2\%) were low grade tumors and $72(75.8 \%)$ were high grade. Chen A found $52 \%$ low grade and $47 \%$ high grade tumors. ${ }^{16}$ Lotan recorded $91 \%$ patients at Ta-TI, $2.4 \% \mathrm{~T} 2-3$, and unrecorded $6.4 \%$ patients out of $157 .{ }^{11}$ Among our patients $74.7 \%$ were diagnosed at stage I (T1) and $25.3 \%$ were stage II (T2) cancers showing late stage diagnosis as compared to other studies.

Tissue NMP 22 was expressed immunohistochemically in $91.6 \%$ of cases and was negative in $8.4 \%$ of cancers. Balci showed that $66.7 \%$ of his patients were positive for NMP22 and $33.3 \%$ were negative. ${ }^{17}$ In the present group, strong intensity of staining was observed in $44.2 \%$, moderate in $20 \%$, weak in $27.4 \%$ and none in $8.4 \%$ of cases. Similarly, Tsai et al. reported low intensity (0-150 score) in $21.74 \%$ and high (151-300 score) in $78.26 \%$ of patient population, $n=92 .{ }^{10}$ In this study, association of NMP22 expression with gender showed that $22 / 26(84.6 \%)$ males and $4 / 26(15.3 \%)$ females were weak positive for NMP22. 18/19 (94.7\%) exhibited moderate positivity in males and $1 / 19$ $(5.26 \%)$ in females. Strong expression was noted in $37 / 42$ (88.1\%) males and 5/42 (11.9\%) females. Eight out of 95 cases were negative out of which 6/8 (75\%) were males and $2 / 8$ (25\%) were females. Many studies found no correlation with gender and NMP22. ${ }^{10,18}$ However, our findings support positive relationship between expression and male gender similar to the findings of other studies, in urine samples. ${ }^{13,19}$

The intensity of NMP22 staining showed positive correlation with histologic grade exhibiting strong NMP22 expression in 
$58.3 \%$ of high grade BC, $23.6 \%$ moderate and $11.1 \%$ weak expression. In low grade tumors no biopsy expressed strong expression. Moderate intensity was observed in $8.69 \%$ and weak in $78.26 \%$ of tumors. Many of the international studies confirm these findings. ${ }^{14,18,19}$ Since urinary detection is not much reliable in predicting grade of tumor, $\mathrm{IHC}$ is superior to urinary tests. $^{18-20}$

Association of expression intensity of NMP22 with stage of tumor revealed that $40.8 \%$ of stage I tumors exhibited strong staining, $16.9 \%$ moderate and $32.3 \%$ weak staining pattern. However, in stage II tumors $54.2 \%$ showed strong, $29.2 \%$ moderate and $12.5 \%$ weak NMP 22 expression.

We conclude that as the stage increases, there is mild increase in expression intensity of NMP22. Although this finding is less significant $(p=0.148)$ than grade but it is not a negligible finding. Most studies have identified similar findings between these two variables except Szymanska B, who documented strong positive association. ${ }^{18,19,20}$

\section{CONCLUSION}

NMP 22 is highly expressed in urinary bladder carcinoma and in male patients. It has strong association with grade; and weak association with stage of tumor. Immunohistochemical expression of this protein can help in better detection and prognostic prediction of bladder carcinoma.

\section{FUNDING:}

This study was funded by King Edward Medical University, Lahore, Pakistan.

\section{ETHICALAPPROVAL:}

Ethical approval was obtained for this study from Institutional Review Board of King Edward Medical University, Lahore, via letterNo. 2069/RC/KEMU dated 23 October 2019.

\section{PATIENTS' CONSENT:}

Informed consents were obtained from patients to publish the data concerning this study.

\section{CONFLICT OF INTEREST:}

Authors declared no conflict of interest.

\section{AUTHORS' CONTRIBUTION:}

QM: Data collection.

SQ: Manuscriptwriting

SA: Data interpretation

MAK: Data analysis.

UA: Data collection

\section{REFERENCES}

1. Sarwar MR, Saqib A. Cancer prevalence, incidence and mortality rates in Pakistan in 2012. Cogent Medicine 2017; 4:1. doi.org/10.1080/2331205X.2017.1288773

2. http://www.cancer.org/cancer/bladder-cancer/about/key-sta tistics.html. Jan2020.

3. Tuna B, Yorukoglu K, Duzcan E, Sen S, Nese N, Sarisk B, et al. Histological grading of papillary urothelial neoplasm:
Impact of combined grading (two numbered grading system) on reproducibility. Virchows Arch 2011; 458(6): 659-64. doi: 10.1007/s00428-011-1072-3.

4. Sharma P, Kini H, Pai RR, Sahu KK, Kini J. Study of the reproducibility of 2004 world health organization classification of urothelial neoplasms. Indian J Pathol Microbiol 2015; 58(1):59-61. doi: 10.4103/0377-4929. 151189.

5. Zhou B, Guo R. Integrative analysis of genomic and clinical data reveals intrinsic characteristics of bladder urothelial carcinoma progression. Genes (Basel). 2019; 10(6): pii:E464. doi: 10.3390/genes10060464.

6. Bernardo C, Eriksson P, Marzouka NA, Liedberg F, Sjödahl $G$, Höglund M. Molecular pathology of the luminal class of urothelial tumors. J Pathol 2019; 249(3):308-18.doi: 10.1002/path.5318. Epub 2019 Aug 27.

7. Breen V, Kasabov N, Kamat AM, Jacobson E, Suttie JM, O'Sullivan $\mathrm{PJ}_{L}$ et al. A holistic comparative analysis of diagnostic tests for urothelial carcinoma: A study of cxbladder detect, urovysion ${ }^{\circledR}$ FISH, NMP $22 \AA$ and cytology based on imputation of multiple datasets. BMC Med Res Methodol. 2015; 15:45. doi: 10.1186/s12874-015-0036-8.

8. Makimoto G, Nishimori $H$, Kondo R, Yanai $H$, Sugimoto $M$, Oda $N$, et al. Successful treatment of metastatic urothelial carcinoma after accurate diagnosis by immunohistochemistry. Acta Med Okayama 2019; 73(3):279-84. doi: 10.1186/s12874-015-0036-8.

9. Abdel-Rahman O. Bladder cancer mortality after a diagnosis of nonmuscle-invasive bladder carcinoma. Future Oncol 2019; 15(19):2267-75. doi: 10.2217/fon-2018-0861.

10. Tsai C, Chen Y, Chang Y, Hsueh C, Liu C, Chang Y.et al. Systematic verification of bladder cancer-associated tissue protein biomarker candidates in clinical urine specimens. Oncotarget 2018; 9(56): 30731-30747. doi: 10.18632/oncotarget.24578.

11. Lotan $Y, O^{\prime}$ Sullivan P, Raman JD, Shariat SF, Kavalieris L, Frampton $C_{1}$ et al. Clinical comparison of noninvasive urine tests for ruling out recurrent urothelial carcinoma. Urol Oncol 2017; 35(8):531.e15-531.e22. doi: 10.1016/j. urolonc.2017.03.008.

12. Liang Q, Zhang G, Li W, Wang J, Sheng S. Comparison of the diagnostic performance of fluorescence in situ hybridization (FISH), nuclear matrix protein 22 (NMP22), and their combination model in bladder carcinoma detection: a systematic review and meta-analysis. Onco Targets Ther 2018; 12:349-58. doi: 10.2147/OTT.S186065.

13. Lotan Y, Svatek RS, Krabbe LM, Xylinas E, Klatte T, Shariat SF. Prospective external validation of a bladder cancer detection model. J Urol 2014; 192(5):1343-8. doi: 10.1016/j.juro.2014.05.087.

14. Coskuner E, Cevik I, Ozkan A, Dillioglugil O, Akdas A. In the cystoscopic follow-up of non-muscle-invasive transitional cell carcinoma, NMP-22 works for high grades, but unreliable in low grades and upper urinary tract tumors. Int Urol Nephrol 2012; 44(3):793-8. doi: 10.1007/s11255-0120144-x.

15. Bier S, Hennenlotter J, Esser M, Mohrhardt S, Rausch S, Schwentner $C_{L}$ et al. Performance of urinary markers for detection of upper tract urothelial carcinoma: Is upper tract 
urine more accurate than urine from the bladder? Dis Markers 2018; 30 2018:5823870. doi: 10.1155/2018/ 5823870 .

16. Chen A, Fu G, Xu Z, Sun Y, Chen X, Cheng $\mathrm{KS}_{\perp}$ et al. Detection of urothelial bladder carcinoma via microfluidic immunoassay and single-cell DNA copy-number alteration analysis of captured urinary-exfoliated tumor cells. Cancer Res 2018; 78(14):4073-4085. doi: 10.1158/0008-5472. CAN-17-2615.

17. Balci M, Tuncel A, Guzel O, Aslan Y, Sezgin T, Bilgin O, et al. Use of the nuclear matrix protein 22 Bladder Chek test ${ }^{\mathrm{TM}}$ in the diagnosis of residual urothelial cancer before a second transurethral resection of bladder cancer. Int Urol Nephrol 2015; 47(3):473-7. doi: 10.1007/s11255-015-0921-4.
18. Szymańska B, Sawicka E, Guzik A, Zdrojowy R, Długosz A. The diagnostic value of nuclear matrix proteins in bladder cancer in the aspect of environmental risk from carcinogens. BioMed Res Internat 2017; 2017:9643139

19. Zhou BF, Wei JH, Chen ZH, Dong P, Lai YR, Fang $Y_{L}$ et al. Identification and validation of AIB1 and EIF5A2 for noninvasive detection of bladder cancer in urine samples. Oncotarget 2016; 7(27):41703-714. doi: 10.18632/ oncotarget.9406.

20. Önal B, Han Ü, Yilmaz S, Köybasioglu F, Altuğ U. The use of urinary nuclear matrix protein 22 (NMP22) as a diagnostic adjunct to urine cytology for monitoring of recurrent bladder cancer--institutional experience and review. Diagn Cytopathol 2015; 43(4):307-14. doi: 10.1002/dc.23239. 